\title{
La carga precoz de los implantes GMI con conexión interna y superficie arenada y grabada
}

\section{Early loading of GMI implants with internal connection and sandblasted- acid-etched surface}

\author{
VELASCO ORTEGA E* \\ MONSALVE GUIL $\mathbf{L} * *$ \\ MATOS GARRIDO N*** \\ JIMÉNEZ GUERRA A $* * *$ \\ GARCÍA IMÉNDEZ A** \\ MEDEL SOTERAS R** \\ ORTIZ GARCÍA I $* * *$ \\ ESPAÑ̃ LÓPEZ $A * * *$
}

\begin{abstract}
Velasco Ortega E, Monsalve Guil L, Matos Garrido N, Jiménez Guerra A, García Méndez A, Medel Soteras R, Ortiz García I, España López A. La carga precoz de los implantes GMI con conexión interna y superficie arenada y grabada. Av Periodon Implantol. 2014; 26, 2: 67-75.
\end{abstract}

\section{RESUMEN}

Introducción: La implantología oral representa en la actualidad, una modalidad terapéutica odontológica en los pacientes con pérdida dental total y parcial. El estudio muestra la evaluación de los pacientes tratados mediante carga precoz de implantes de titanio con conexión interna y superficie arenada y grabada.

Métodos: 39 pacientes con pérdidas dentales fueron tratados con implantes con conexión interna y superficie arenada y grabada Frontier GMI@. Los implantes fueron cargados funcionalmente tras un periodo de tiempo de 6 semanas en la mandíbula y 8 semanas en el maxilar superior. Los hallazgos clínicos (implantológicos y prostodóncicos) se han seguido durante 1 año.

Resultados: 11 implantes fueron insertados en ambos maxilares (50 en el maxilar superior y 61 en la mandíbula) para su rehabilitación prostodóncica. 44 implantes fueron insertados en el sector anterior y 67 implantes en el sector posterior. Después de 1 año de seguimiento clínico, los resultados indican una supervivencia y éxito de los implantes del 99,1\%. Durante el periodo de cicatrización libre de carga funcional, se perdió 1 implante por movilidad. Como complicaciones tardías hubo un caso de periimplantitis. Las restauraciones prostodóncicas incluyeron 30 coronas unitarias, 15 puentes fijos, 5 sobredentaduras con bolas y 2 rehabilitaciones totales fijas.

Conclusiones: Los hallazgos clínicos del presente estudio indican que la rehabilitación prostodóncica mediante la inserción de implantes de titanio con conexión interna y superficie arenada + grabada, y cargados precozmente, representa una terapéutica odontológica con éxito elevado.

PALABRAS CLAVE: Implantes dentales, superficie arenada y grabada, conexión interna, carga precoz, oseointegración, implantología oral.

\section{SUMMARY}

Introduction: Today, oral implantology constitute a therapeutic modality in the prosthodontic treatment of patients with partial and total tooth loss. This study reports the evaluation of patients treated by early loading of titanium implants with internal connection and sandblasted-acidetched surface.

Profesor titular de Odontología Integrada de Adultos. Director del Máster de Implantología Oral. Facultad de Odontología. Universidad de Sevilla.

** Profesor asociado de Odontología Integrada de Adultos. Profesor del Máster de Implantología Oral. Facultad de Odontología. Universidad de Sevilla.

*** Profesor colaborador clínico de Odontología Integrada de Adultos. Profesor del Máster de Implantología Oral. Facultad de Odontología. Universidad de Sevilla. 
Methods: 39 patients with tooth loss were treated with Frontier GMI $®$ sandblasted and acid-etched surface implants. Implants were loaded after a healing free-loading period of 6 weeks (mandible) and 8 weeks (maxilla). Clinical findings (implants and prosthodontics) were followed during l year. Results: 111 implants were inserted (50 maxillary, and 61 mandibular) for prosthodontic rehabilitation. 44 implants were inserted in anterior sites and 67 in posterior sites. After l-year follow-up, clinical results indicate a survival and success rate of implants of $99.1 \%$. One implant were lost during the healing period by mobility. A case of periimplantitis were reported as late complication after functional loading. Prosthodontic restorations included 30 single crowns, 15 fixed bridges, 5 balloverdentures and 2 fixed totally rehabilitation.

Conclusions: Clinical results of this study indicate that prosthodontic rehabilitation supported by internal connection and sandblasted and etched-titanium implants and loaded early can be a successful dental treatment.

KEY WORDS: Dental implants, sandblasted and acid-etched surface, internal connection, early loading, osseointegration, implant dentistry.

Fecha de recepción: 15 de julio de 2013.

Fecha de aceptación: 8 de octubre de 2013.

\section{INTRODUCCIÓN}

La implantología oral ha representado un impacto muy importante en la profesión dental al representar un técnica terapéutica predecible y de éxito en el tratamiento de los pacientes con edentulismo total y parcial. En este sentido, los avances en el diagnóstico integral del paciente, en la planificación de tratamiento, en las técnicas quirúrgicas básicas y complejas, así como en las diversas opciones prostodóncicas (coronas, puentes, rehabilitaciones fijas y sobredentaduras) han desarrollado la implantología oral hasta convertirla en una terapéutica odontológica cotidiana (1-6).

Las indicaciones iniciales de los implantes dentales oseointegrados y la experiencia clínica acumulada fue el resultado del tratamiento en los pacientes edéntulos totales mediante la inserción de algunos implantes y la rehabilitación con una prótesis completa híbrida atornillada (7). Posteriormente, el tratamiento implantológico ha sido aplicado a los pacientes con pérdida dental parcial y unitaria, demostrando ser una alternativa con éxito a las prótesis parciales removibles y fijas, tanto en el maxilar como en la mandíbula (4-6).

La oseointegración como fenómeno biológico fue originalmente descrita en el protocolo clásico de Branemark, según una técnica quirúrgica de 2 fases y un periodo de cicatrización libre de carga funcional de 3-6 meses con implantes de titanio roscados y de superficie lisa (7). En este sentido, se preconizaba que la carga prematura po- día interferir la unión del hueso a la superficie de titanio del implante y provocar una encapsulación fibrosa (8). Sin embargo, la investigación experimental y clínica posterior ha demostrado que mediante diversos protocolos quirúrgicos y prostodóncicos la carga precoz per se no impide la oseointegración, siempre que se reduzcan los micromovimientos durante la cicatrización con una mejor estabilidad primaria de los implantes (8).

Los avances en el diseño macroscópico de los implantes ha impulsado la introducción de nuevos sistemas con una estructura macroscópica más autorroscante y que proporcione una mayor estabilidad primaria (9). Además, ciertas modificaciones macroscópicas en los implantes, especialmente en su área cervical, además de incrementar su estabilidad primaria puede ayudar a mantener una mejor salud de los tejidos periimplantarios óseos y conservar más favorablemente el nivel de hueso marginal (10).

Además, el desarrollo de superficies tratadas de los implantes indica que una mayor rugosidad (ej.: arenado y grabado ácido) favorece la oseointegración y reduce el tiempo de cicatrización, lo que posibilita la carga precoz de los implantes mediante la correspondiente restauración prostodóncica (11-15).

El objetivo del presente estudio era la valoración clínica y el seguimiento del tratamiento con implantes GMI con superficie arenada y grabada y cargados precozmente. 


\section{PACIENTES Y MÉTODOS}

El presente estudio fue realizado por profesores de las unidades docentes de Odontología Integrada de Adultos y del Máster de Implantología Oral de la Facultad de Odontología de la Universidad de Sevilla.

Previamente a la realización del estudio, aquellos pacientes que padecían trastornos sistémicos graves que podían comprometer la oseointegración fueron excluidos del estudio. Los pacientes seleccionados eran adultos, de ambos sexos. Todos los pacientes presentaban pérdidas dentales parciales o totales. Todos los pacientes seleccionados en el estudio fueron informados del protocolo del tratamiento con implantes, de los aspectos quirúrgicos y prostodóncicos, temporalización del tratamiento y seguimiento, así como de la posibilidad de la existencia de complicaciones y pérdida de implantes. Los pacientes autorizaron el tratamiento implantológico mediante un consentimiento informado. Antes del tratamiento, todos los pacientes fueron evaluados radiológicamente, con una ortopantomografía y en los casos necesarios con una tomografía computarizada de haz cónico.

Los criterios de éxito y supervivencia de los implantes fueron los recomendados por van Steenberghe et al (16). En este sentido, la supervivencia fue definida como la presencia permanente de los implantes en su localización original aunque no tengan valor clínico o cause efectos adversos. Los criterios de éxito de los implantes se expresan en la Tabla 1.

\section{Cirugía}

Una hora antes de la cirugía, los pacientes comenzaron un régimen antibiótico preventivo (amoxicilina + clavulánico) durante una semana. Todos los pacientes recibieron anestesia local. Todos los implantes utilizados en el presente estudio eran Frontier ${ }^{\circledR}$ (Global

\begin{tabular}{|l|c|c|c|}
\hline \multicolumn{4}{|c|}{ TABLA 1.- DISTRIBUCIÓN DE LOS } \\
IMPLANTES SEGÚN SU LOCALIZACIÓN \\
\hline \multirow{3}{*}{} & \multicolumn{3}{|c|}{ Implantes } \\
\cline { 2 - 4 } & Anterior & Posterior & Total \\
\hline Maxilar & 21 & 29 & 50 \\
Mandíbula & 23 & 38 & 61 \\
\hline Total & 44 & 67 & 111 \\
\hline
\end{tabular}

Medical Implants, Barcelona, España) roscados de conexión interna y de superficie arenada y grabada. Todos los implantes fueron estables después de la inserción. La preparación del lecho y la inserción de los implantes se realizó según el protocolo estandarizado convencional con fresas consecutivas de menor a mayor diámetro, a una velocidad constante de $800 \mathrm{rpm}$. A la semana se retiraron las suturas y a los pacientes se les recomendó el enjuague diario con clorhexidina durante los primeros 30 días (Figuras 1-4).

\section{Prostodoncia}

A las 6 semanas (en la mandíbula) y a las 8 semanas (en el maxilar) después de la inserción de los implan-

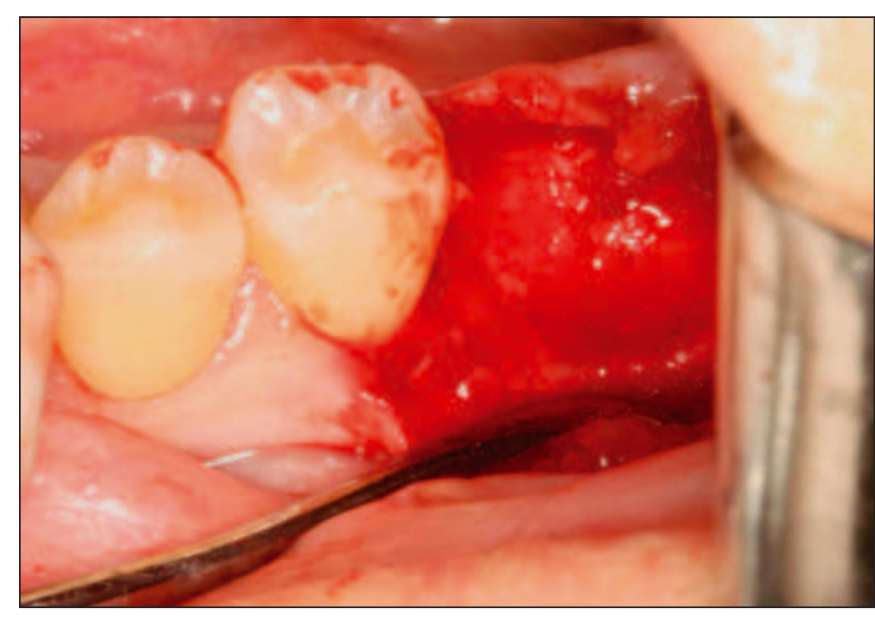

Fig. 1: Cirugía con colgajo para inserción de implante en sustitución del primer molar inferior izquierdo.

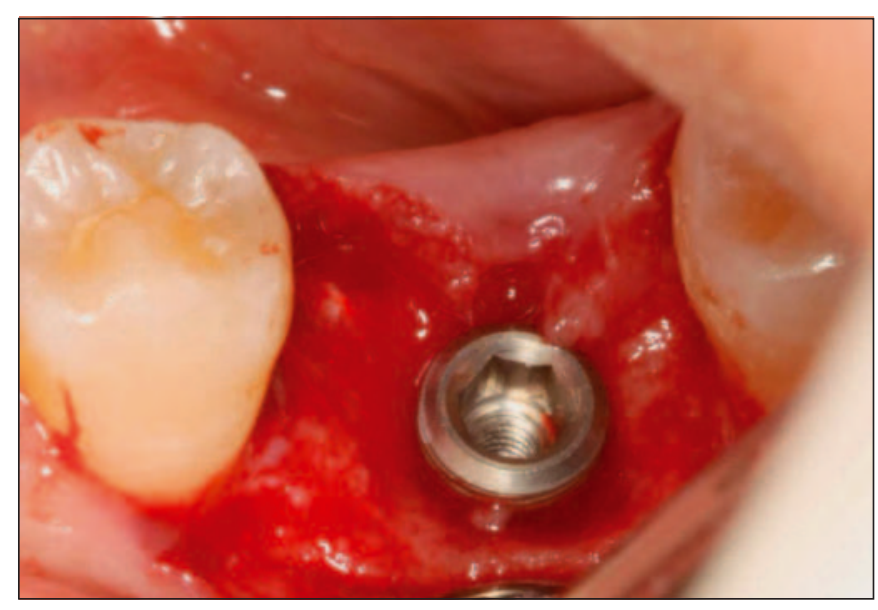

Fig. 2: Aspecto clínico del implante de conexión interna insertado. 


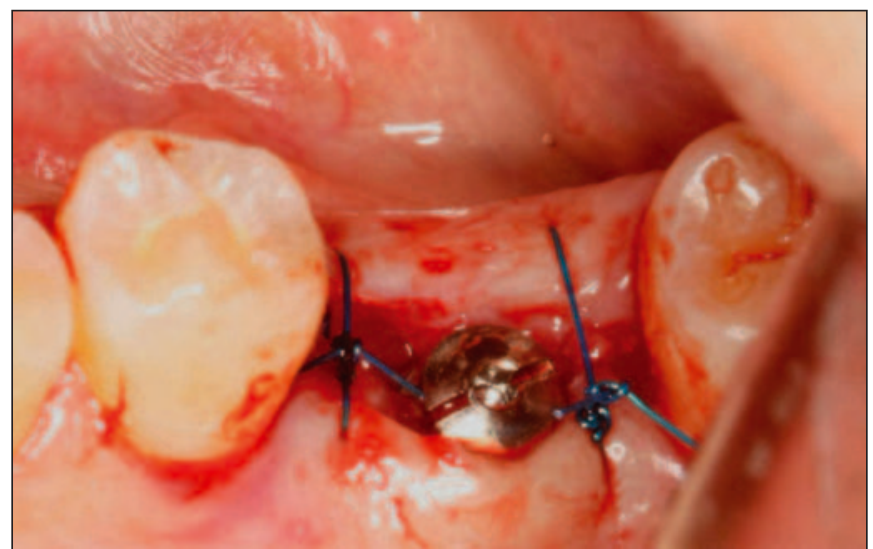

Fig. 3: Aspecto clínico de la sutura alrededor del implante.

tes, se realizó la carga precoz funcional de los implantes mediante la colocación de las correspondientes prótesis implantosoportadas (Figuras 5 y 6 ). El tiempo transcurrido de seguimiento clínico desde la carga funcional de los implantes fue de 12 meses.

\section{Análisis estadístico}

Se realizó una estadística descriptiva de los hallazgos clínicos del estudio, con referencia a las variables de- mográficas de los pacientes, la supervivencia, el éxito, las complicaciones y pérdidas de los implantes así como de las restauraciones prostodóncicas realizadas.

\section{RESULTADOS}

\section{Pacientes}

Participaron en el estudio 39 pacientes con pérdidas dentales ( 21 hombres y 18 mujeres), con una edad media de 45,3 años (rango: 25-73 años); 6 pacientes $(15,4 \%)$ eran edéntulos totales; 7 pacientes $(18 \%)$ presentaban alguna enfermedad sistémica (hipertensión arterial, diabetes) aunque controlada; 8 pacientes $(20,5 \%)$ eran fumadores.

\section{Implantes}

Se insertaron un total de 111 implantes Frontier GMI@ en los correspondientes 39 pacientes; 50 implantes (45\%) fueron insertados en el maxilar superior y 61 implantes (55\%), en la mandíbula; 44 implantes $(39,6 \%)$ fueron insertados en el sector anterior (sustitución de incisivos y caninos) y 67 implantes $(60,4 \%)$, en el sector posterior (sustitución de premolares y molares) (Tabla 1).

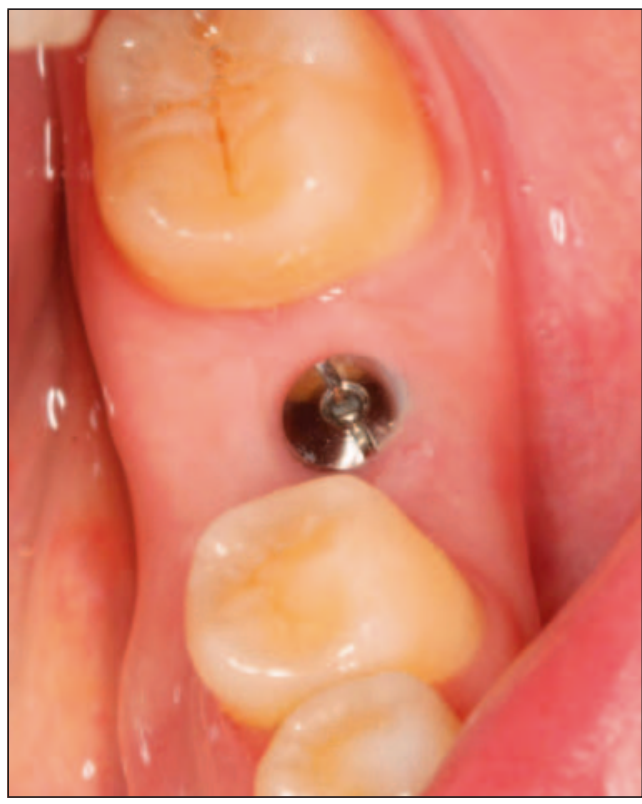

Fig. 4: Aspecto clínico a las 6 semanas. Se observa el buen estado de los tejidos blandos alrededor del tornillo de cicatrización.

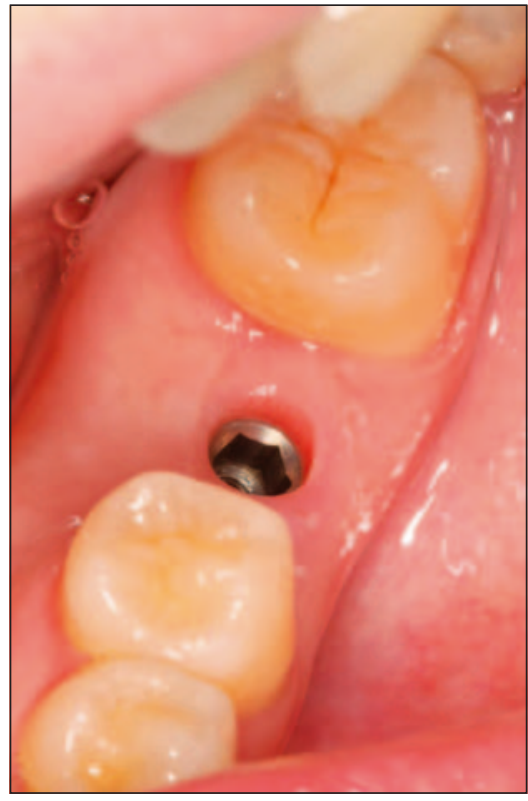

Fig. 5: Aspecto clínico a las 6 semanas. Se observa el buen estado de los tejidos blandos alrededor del implante.

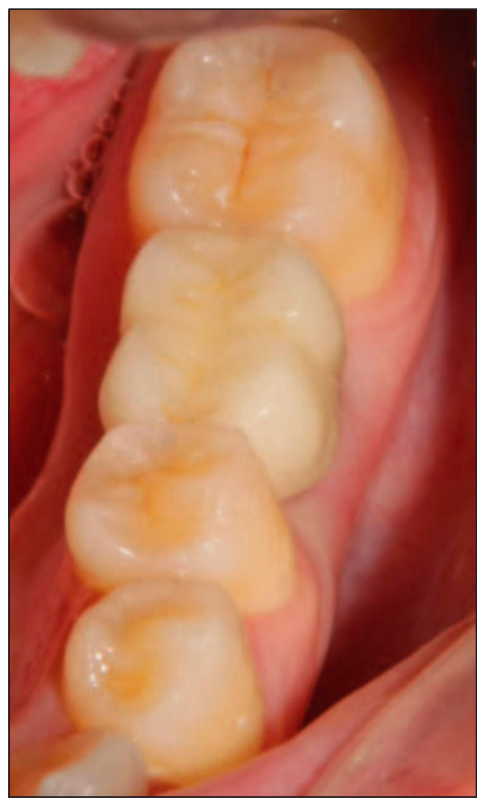

Fig. 6: Aspecto clínico de la corona cementada sobre el implante de conexión interna. 
Cincuenta y nueve implantes $(53,2 \%)$ fueron insertados de forma sumergida (2 cirugías) mientras que 52 implantes $(46,8 \%)$ se insertaron de forma no sumergida (una cirugía); 102 implantes $(92,8 \%)$ fueron insertados mediante cirugía implantológica básica, mientras que 8 implantes $(7,2 \%)$ fueron insertados mediante técnicas complejas (osteótomos, injertos, regeneración ósea guiada).

De los implantes utilizados, 6 fueron de $3,3 \mathrm{~mm}$ de diámetro; 45 de $3,75 \mathrm{~mm}$ de diámetro; 42 fueron de 4,25 $\mathrm{mm}$ de diámetro y 18 de $4,75 \mathrm{~mm}$. Con respecto a la longitud, fueron insertados 83 implantes de $11,5 \mathrm{~mm}$, 26 de $10 \mathrm{~mm}$ y 2 de $8 \mathrm{~mm}$ (Tabla 2). Todos los implantes fueron insertados de forma diferida, tras un periodo mínimo de 6 meses después de la extracción dental.

Como complicaciones inmediatas, un implante $(0,9 \%)$ presentó movilidad a la exploración clínica durante la fase de cicatrización libre de carga funcional y fue extraído. Como complicaciones tardías hubo un caso de periimplantitis que fue tratada con regeneración ósea. No hubo ninguna pérdida de implante después de la carga funcional mediante su restauración prostodóncica correspondiente durante el año de seguimiento clínico.

\section{Prótesis implantosoportadas}

Se realizaron 52 prótesis en los 39 pacientes. 30 coronas unitarias $(57,7 \%), 15$ prótesis parciales fijas $(28,9 \%)$, 5 sobredentaduras retenidas por bolas $(9,6 \%)$ y 2 rehabilitaciones totales fijas $(3,8 \%)$. De las 47 prótesis fijas, $28(59,6 \%)$ fueron cementadas y el resto, $19(40,4 \%)$ fueron atornilladas.

\section{TABLA 2.- DISTRIBUCIÓN DE LOS IMPLANTES SEGÚN SU DIAMETRO Y LONGITUD}

\begin{tabular}{|l|c|r|r|r|}
\hline \multirow{2}{*}{ Diámetro } & \multicolumn{3}{|c|}{ Longitud } & \multirow{2}{*}{ Total } \\
\cline { 2 - 4 } & $\mathbf{8 ~ \mathbf { ~ m m }}$ & $\mathbf{1 0} \mathbf{~ m m}$ & $\mathbf{1 1 , 5} \mathbf{~ m m}$ & \\
\hline $3,30 \mathrm{~mm}$ & - & $2 \mathrm{impl}$. & $4 \mathrm{impl}$. & $6 \mathrm{impl}$. \\
$3,75 \mathrm{~mm}$ & - & $8 \mathrm{impl}$. & $37 \mathrm{impl}$. & $45 \mathrm{impl}$. \\
$4,25 \mathrm{~mm}$ & - & $7 \mathrm{impl}$. & $35 \mathrm{impl}$. & $42 \mathrm{impl}$. \\
$4,75 \mathrm{~mm}$ & $2 \mathrm{impl}$. & $9 \mathrm{impl}$. & $7 \mathrm{impl}$. & $18 \mathrm{impl}$. \\
\hline Total & $2 \mathrm{impl}$. & $26 \mathrm{impl}$. & $83 \mathrm{impl}$. & $111 \mathrm{impl}$. \\
\hline
\end{tabular}

\section{DISCUSIÓN}

El objetivo fundamental del presente estudio era obtener una buena oseointegración de los implantes con superficie arenada y grabada y conseguir unos buenos resultados funcionales y estéticos mediante la carga con sus correspondientes prótesis. En este sentido, los hallazgos clínicos del presente estudio, indican una tasa de éxito del 99,1\%, lo que confirma la eficacia de la carga precoz de los implantes dentales utilizados con un diseño macroscópico adecuado y una superficie arenada y grabada, insertados mediante una o dos cirugías, en los pacientes con pérdidas dentales parciales y totales. En el presente trabajo, se presentan los resultados a 1 año, aunque el objetivo es el seguimiento clínico de los pacientes a largo plazo.

En el presente estudio, el 53,2\% de los implantes fueron insertados de forma sumergida (2 fases) mientras que el $46,8 \%$ de los implantes se insertaron de forma no sumergida (una fase), demostrando que ambas técnicas quirúrgicas presentaron una elevada tasa de éxito $(98,3 \%$ vs $100 \%)$. Aunque en el protocolo clásico del grupo sueco de Branemark (7) se recomendaba como un requisito para conseguir la oseointegración de los implantes, la realización de 2 cirugías; sin embargo la experiencia acumulada, fundamentalmente, por la escuela suiza, demuestra que la técnica no sumergida (1 cirugía) proporciona, así mismo, una buena respuesta tisular ósea y de los tejidos blandos $(17,18)$. La valoración clínica y el seguimiento a largo plazo de ambas técnicas quirúrgicas (sumergida, 2 fases y no sumergida, 1 fase) indican que los resultados funcionales y estéticos con los diferentes sistemas de implantes son muy aceptables $(19,20)$. De hecho, se han comparado los resultados clínicos de los implantes Branemark insertados con ambos procedimientos ( 1 ó 2 fases quirúrgicas) sin diferencias de supervivencia y éxito, lo que indica que ambas técnicas son igualmente predecibles (21).

El presente estudio muestra los hallazgos clínicos de la utilización de los implantes en ambos maxilares, en este sentido, de los 111 implantes insertados, el 55\% fue insertado en la mandíbula y el $45 \%$ en el maxilar superior. Esta distinción se basa, fundamentalmente, en el hecho de que la experiencia acumulada indica que las tasas de supervivencia y éxito de los implantes es menor en el maxilar superior que en la mandíbula. Sin embargo, en el presente estudio la pérdida de implantes fue mínima y no tenía relación con la localización maxilar o mandibular. Además, en el presente estudio, la mayoría de los pacientes presenta- 
ban pérdidas unitarias o parciales y la presencia de dientes naturales adyacentes a los implantes pueden ayudar a impedir o retrasar la reabsorción alveolar, y pueden favorecer una protección oclusal comparados con los implantes insertados para la rehabilitación de tramos edéntulos totales (22-24).

El presente estudio describe los resultados clínicos de la utilización de los implantes en sectores anteriores (incisivos y caninos) y posteriores (premolares y molares). En este sentido, de los 111 implantes insertados, el 60,4\% fueron insertados en el sector posterior y el $39,6 \%$ en el sector anterior. El sector posterior representa el área ideal para valorar el éxito de los implantes porque son sometidos a mayores fuerzas masticatorias y las cargas oclusales y parafuncionales se concentran más en esta región $(15,22,23)$. En este sentido, los implantes de diámetro más ancho pueden suponer una alternativa ya que consiguen una mayor interfase titanio-hueso y son más resistentes que los implantes estándares, además de que también favorecen la estética con un buen perfil de emergencia (25). En el sector anterior, el compromiso estético es muy importante ya que los implantes pueden sustituir dientes anteriores que se han perdido por traumatismos, fracasos endodóncicos o enfermedad periodontal avanzada. En estos casos, los implantes representan la mejor terapéutica actual porque las coronas implantosoportadas ofrecen una buena función y estética, y la respuesta del paciente al tratamiento es muy positiva (26).

En el presente estudio, los implantes presentaban longitudes de entre 8 y 11,5 mm y el diámetro oscilaba entre los 3,3 mm y los 4,75 mm, aunque la mayoría de los implantes tenían características macroscópicas estándar (Tabla 2). En este sentido, la estructura macroscópica de los implantes está también relacionada con el éxito del tratamiento $(9,10)$. De hecho, la longitud de los implantes puede influir en el éxito de los tratamientos, es decir, a mayor longitud del implante dental, existirá una mayor superficie de contacto, lo que permitirá aumentar el ratio de contacto entre hueso e implante, por ejemplo, un implante de $10 \mathrm{~mm}$ aumenta un $30 \%$ su área de contacto respecto a un implante de $7 \mathrm{~mm}$ (27). También influye en la oseointegración el diámetro del implante dental. Al igual que la longitud, un mayor diámetro implantario asegura una mayor superficie de contacto con el hueso, lo que asegurará una mejor respuesta biomecánica. Los estudios señalan que el aumento de diámetro influye más positivamente en la integración del implante que el aumento de la longitud del mismo, pero no es ca- paz de compensar la falta de longitud de los implantes cortos (27).

Además de la calidad ósea y la técnica quirúrgica, el diseño macroscópico de los implantes es también muy importante para conseguir una buena estabilidad primaria después de la cirugía (9). La capacidad autorroscante y el diseño cónico puede proporcionar un buen anclaje en el hueso alveolar y obtener un buen contacto hueso-implante después del fresado (9). La incorporación de espiras de menor tamaño en el área cervical, como presentan los implantes insertados en el presente estudio, ha demostrado un incremento de la capacidad para resistir fuerzas axiales y ayuda a preservar el hueso marginal periimplantario (10).

El presente estudio valora los resultados clínicos de la utilización de implantes con una superficie arenada y grabada. La experiencia con implantes de superficie rugosa en el tratamiento de las pérdidas dentales confirma un elevado éxito de esta terapéutica implantológica con resultados a medio y largo plazo altamente predecibles, con este tipo de superficie $(28,29)$. Es evidente que la terapéutica con implantes dentales depende directamente del contacto entre el hueso y la superficie del implante (7). Así, la incorporación de superficies tratadas que incrementen su rugosidad puede favorecer la unión entre la superficie del implante y el hueso, mejorando los resultados clínicos implantológicos $(28,29)$. En este sentido, diversos estudios confirman que la oseointegración puede ser facilitada por la utilización de implantes con superficie rugosa por la acción del arenado y grabado con ácidos, lo que demuestra que existe una buena respuesta de los tejidos del huésped, que se manifiesta por una elevada tasa de éxito $(30,31)$. Además, los implantes con superficie arenada y grabada obtiene mejores resultados clínicos que los implantes con superficie pulida en condiciones con pobre calidad de hueso o en técnicas con regeneración ósea con biomateriales ya que la superficie tratada del implante puede optimizar la respuesta biológica del hueso (32).

En el presente estudio, durante el periodo de cicatrización 1 implante presentó movilidad y fue extraído. Estos fracasos precoces puede ser provocados por factores relacionados con el procedimiento quirúrgico como son el sobrecalentamiento del lecho o la falta de estabilidad primaria que impiden una adecuada unión entre la superficie del implante y el hueso (33). Posteriormente, hubo un caso de periimplantitis como complicación tardía que fue tratado con éxito mediante regeneración ósea guiada (34). Después de la fase 
prostodóncica realizada, los resultados demuestran un éxito del $100 \%$ de los implantes durante los 12 meses transcurridos desde la carga funcional precoz.

La carga precoz constituye uno de los aspectos más importantes que se han valorado en el presente estudio. Aunque, al comienzo de la implantología oral se preconizaba un tiempo de cicatrización libre de carga funcional de 3-6 meses, de hecho, en la actualidad la carga precoz representa un avance en el desarrollo de los protocolos implantológicos, ya que al reducir el tiempo de espera, beneficia a los pacientes con diferentes pérdidas dentales (12-15). Los protocolos de carga precoz se han desarrollado porque han mejorado los aspectos quirúrgicos de los implantes. En este sentido, el diseño macroscópico y la superficie rugosa han logrado establecer un buen contacto hueso-implante y una rápida oseointegración (12-15).

Desde un punto de vista prostodóncico, la carga precoz ha sido utilizada en diferentes estudios que incluyen todos los tipos de restauraciones sobre implantes, como son las coronas unitarias, los puentes fijos, las rehabilitaciones totales y las sobredentaduras $(6,13$, $15,17)$. La carga precoz ha mejorado con las nuevas conexiones internas que obtienen una excelente fijación y transmisión de fuerzas de los pilares a los implantes, reduciendo el tiempo de espera funcional $(35,36)$. Estos hallazgos clínicos son confirmados por el presente estudio, donde los implantes utilizados presentaban una conexión interna para su unión a los correspondientes pilares protésicos. La conexión interna ha supuesto un avance importante en los resultados clínicos funcionales y estéticos de las prótesis implantosoportadas ya que mejoran la respuesta biomecánica al estrés, disminuyen la contaminación bacteriana de la unión pilar-implante y proporcionan un buen sellado para conseguir una excelente estética $(35,36)$.

\section{CONCLUSIONES}

La rehabilitación oral con prótesis soportadas con implantes se ha convertido en un tratamiento odontológico cotidiano. Un requisito para el éxito de esta terapéutica es conseguir y mantener la oseointegración de los implantes. En este sentido, la utilización de técnicas quirúrgicas con implantes con un diseño macroscópico adecuado para conseguir una buena estabilidad quirúrgica y de superficie arenada y grabada para conseguir un nivel aceptable de oseointegración, seguidos de la restauración con las prótesis correspondientes mediante protocolos de car- ga precoz representa un tratamiento con éxito que satisface positivamente al paciente mejorando su calidad de vida oral.

\section{BIBLIOGRAFÍA}

1. Jemt T, Stenport V. Implant treatment with fixed prostheses in the edentulous maxilla. Part 1: Implants and biologic response in two patients cohorts restored between 1986 and 1987 and 15 years later. Int J Prosthodont 2011;24: 345-55.

2. Jemt T, Stenport V. Implant treatment with fixed prostheses in the edentulous maxilla. Part 2: prosthetic technique and clinical maintenance in two patient cohorts restored between 1986 and 1987 and 15 years later. Int J Prosthodont 201 1;24:356-62.

3. Attard NJ, Zarb GA. Long-tem treatment outcomes in edentulous patients with implant-fixed prostheses: the Toronto study. Int J Prosthodont 2004;17:417-24.

4. Simonis P, Dufour T, Tenenbaum H. Long-term implant survival and success: a 10-16 year follow-up of nonsubmerged dental implants. Clin Oral Implant Res 2010; 21:772-7.

5. Mertens C, Steveling HG, Stucke K, Pretzl B, MeyerBäumer A. Fixed implant-retained rehabilitation of the edentulous maxilla: 11 -year results of a prospective study. Clin Impl Dent Relat Res 2012;14:816-27.

6. Buser D, Janner SFM, Wittneben JG, Brägger U, Ramseier CA, Salvi GE. 10-year survival and success rates of 511 titanium implants with a sandblasted and acid-etched surface: a retrospective study in 303 partially edentulous patients. Clin Impl Dent Relat Res 2012;14:839-51.

7. Branemark PI, Zarb GA, Albrektsson T (eds.). Tissue integrated prostheses: osseointegration in clinical dentistry. Chicago. Quintessence. 1985.

8. Grandi T, Garuti G, Guazzi P, Tarabini L, Forabosco A. Survival and success rates of immediately and early loaded implants: 12-month results from a multicentric randomized clinical study. J Oral Impl 2012;38:239-49.

9. Elias CN, Rocha FA, Nascimento AL, Coelho PG. Influence of implant shape, surface morphology, surgical technique and bone quality on the primary stability of dental implants.J Mech Behav Biomed Mater 2012;16:169-80. 
10. Kang YI, Lee DW, Park KH, Moon IS. Effect of thread size on the implant neck area: preliminary results at 1 year of function. Clin Oral Impl Res 2012;23:1147-51.

11. Esposito M, Grusovin MG, Willings M, Coulthard P, Worthington HV. The effectiveness of immediate, early and conventional loading of dental implants: a Cochrane systematic review of randomized controlled clinical trials. Int J Oral Maxillofac Implants 2007;22:893-904.

12. Cochran DL, Jackson JM, Bernard JP, Ten Bruggentake CM, Buser D, Taylor TD et al. A 5-year prospective multicenter study of early loaded titanium implants with a sandblasted and acid-etched surface. Int J Oral Maxillofac Implants 201 1;26:1324-32.

13. Lethaus B, Kälber J, Petrin G, Brandstätter A, Wingart D. Early loading of sandblasted and acid-etched titanium implants in the edentulous mandible: a prospective 5year study. Int J Oral Maxillofac Implants 201 1;26:887-92.

14. Mertens C, Steveling HG. Early and immediate loading of titanium implants with fluoride-modified surfaces: results of 5-year prospective study. Clin Oral Impl Res 2011;22:1354-60.

15. Bornstein MM, Wittneben JG, Brägger U, Buser D. Early loading at 21 days of non-submerged titanium implants with a chemically modified sandblasted and acidetched surface: 3-year results of a prospective study in the posterior mandible. J Periodontol 2010;81:809-18.

16. Van Steenberghe D, Quirynen, Naert I. Survival and success rates with oral endosseous implants. En: Lang NP, Karring T, Lindhe J (eds.). Proceedings of the 3rd European Workshop on Periodontology. Implant Dentistry. Berlin: Quintessence. 1999. págs: 242-52.

17. Velasco E, Pérez O, Medel R, Segura JJ, Torres R. Carga precoz de los implantes no sumergidos con superficie grabada con ácidos en sobredentaduras mandibulares. Arch Odontoestomatol 2003;19:308-16.

18. Velasco E, Medel R, Linares D, Monsalve L, Velasco C. Los implantes de titanio con superficie grabada con ácidos. Un seguimiento clínico de 2 años. Av Perio Impl Oral 2004;16:179-86.

19. Lekholm U, Gunne J, Henry P, Higuschi K, Lindén U, Bergström C, van Steenberghe D. Survival of the Branemark implant in partially edentulous jaws: a 10year prospective multicenter study. Int J Oral Maxillofac Implants 1999;14:639-45.
20. Buser D, Mericske-Stern RD, Bernard JP, et al. Long-term evaluation of non-submerged ITI implants. Part 1:8-year life table analysis of a prospective multi-center study with 2359 implants. Clin Oral Impl Res 1997;8:161-72.

21. Collaert B, De Bruyn H. Comparison of Branemark fixture integration and short-term survival using onestage or two-stage surgery in completely and partially edentulous mandibles. Clin Oral Impl Res 1998;9:131-5.

22. Gross MD. Occlusion in implant dentistry. A review of the literature of prosthetic determinants and current concepts. Aust Dent J 2008;53(suppl 1):60-8.

23. Carlsson GE. Dental occlusion : modern concepts and their application in implant prosthodontics. Odontology $200 ; 97: 8-17$.

24. Salvi GE, Brägger U. Mechanical and technical risks in implant dentistry. Int J Oral Maxillofac Implants 2009;24(suppl): 69-85

25. Degidi M, Piatelli A, Iezzi G, Carinci F. Wide-diameter implants: analysis of clinical outcome of 304 fixtures. J Periodontol 2007,78:52-8.

26. Délben JA, Goitao MC, Gennari-Filho H, Assunçao WG, dos Santos DM. Esthetics in implant-supported prostheses. A literature review. J Oral Impl 2012;38:71822.

27. Guan H, van Staden R, Loo YC, Johnson N, Ivanovski S, Meredith $\mathrm{N}$. Influence of bone and dental implant parameters on stress distribution in the mandible: a finit element study. Int J Oral Maxillofac Implants 2009;24: 866-76.

28. Velasco E, Pato J, Segura JJ, Medel R, Poyato M, Lorrio JM. La investigación experimental y la experiencia clínica de las superficies de los implantes dentales. Parte I. Dentum 2009;9:101-7.

29. Velasco E, Pato J, Segura JJ, López J, García A, España A. La investigación experimental y la experiencia clínica de las superficies de los implantes dentales. Parte II. Dentum 2009;9:108-13.

30. Le Guéhennec L, Soueidan A, Layrolle P, Amouriq Y. Surface treatments of titanium dental implants for rapid osseointegration. Dental Materials 2007;23:844-54.

31. Bonfante EA, Marin C, Graanto R, Suzuki M, Hjerppe J, Witek L, Coelho PG. Histologic and biomechanical 
evaluation of alumina-blasted/acid-etched and resorbable blasting media surfaces. J Oral Impl 2012;38:549-56.

32. Pato J, Jiménez A, Monsalve L, Segura JJ, Velasco E. Regeneración ósea guiada con implante unitario con nanosuperficie y betafosfato tricálcico. Av Perio Impl Oral 2010;22:127-34.

33. Berglundh T, Persson L, Klinge B. A systematic review of the incidence of biological and technical complications in implant dentistry reported in prospective longitudinal studies of at least 5 years. J Clin Periodontol 2002;29: 197-212.

34. Claffey N, Clarke E, Polyzois I, Renvert S. Surgical treatment of periimplantitis. J Clin Periodontol 2008; 35(suppl.8): 316-32.
35. Finger IM, Castellon P, Block M, Elian N. The evolution of external and internal implant/abutment connections. Pract Proced Aesthet Dent 2003; 15: 625-32.

36. Asvanund P, Morgano SM. Photoelastic stress analysis of external versus internal implant-abutment connections. J Prosthet Dent 201 1;106: 266-71.

\section{CORRESPONDENCIA}

Dr. Eugenio Velasco Ortega

Facultad de Odontología

Avicena, s/n

41009 Sevilla

Correo electrónico: evelasco@us.es 